\title{
The influence of bile salt on the chemotherapeutic response of docetaxel- loaded thermosensitive nanomicelles
}

This article was published in the following Dove Press journal:

International Journal of Nanomedicine

8 August 2014

Number of times this article has been viewed

\author{
Dong Wuk Kim ${ }^{1, *}$ \\ Thiruganesh Ramasamy ${ }^{2, *}$ \\ Ju Yeon $\mathrm{Choi}^{2}$ \\ Jeong Hwan Kim² \\ Chul Soon Yong ${ }^{2}$ \\ Jong Oh $\mathrm{Kim}^{2}$ \\ Han-Gon Choi' \\ 'College of Pharmacy, Institute \\ of Pharmaceutical Science and \\ Technology, Hanyang University, \\ Ansan, South Korea; ${ }^{2}$ College \\ of Pharmacy, Yeungnam University, \\ Gyongsan, South Korea
}

*These two authors contributed equally to this work
Correspondence: Han-Gon Choi

College of Pharmacy, Institute

of Pharmaceutical Science and

Technology, Hanyang University,

55, Hanyangdaehak-ro, Sangnok-gu,

Ansan 426-79I, South Korea

Tel +82 3। 4005802

Fax +82 3I 4005958

Email hangon@hanyang.ac.kr

Jong Oh Kim

College of Pharmacy, Yeungnam

University, 2I4-I, Dae-Dong,

Gyongsan, 7I2-749, South Korea

$\mathrm{Tel}+82538102813$

Fax +82 538104654

Email jongohkim@yu.ac.kr
Abstract: The primary aim of this work was to investigate the potential of bile salt, sodium taurocholate (NaTC), in improving the bioavailability and anti-tumor efficacy of docetaxel (DCT) upon rectal administration. Poloxamer-based nanomicelles with thermosensitive and mucoadhesive properties were prepared using the cold method. The optimized nanomicellar formulation was evaluated in terms of physicochemical and viscoelastic parameters. Nanomicelles containing bile salt maintained sufficient gelation strength $\left(234 \times 10^{2} \mathrm{mPa} \cdot \mathrm{s}\right)$ and mucoadhesive force $\left(17.3 \times 10^{2} \mathrm{dyne} / \mathrm{cm}^{2}\right)$ to be retained in the upper part of the rectum. They significantly enhanced the DCT internalization across the rectal mucosa and showed a high plasma level during the first 4 hours of the study period, compared to nanomicelles with no bile salt. As a result, a slightly higher rectal bioavailability of $\sim 33 \%$ was observed in nanomicelles containing bile salt, compared to $\sim 28 \%$ from the latter system. The higher pharmacokinetic parameters for rectally administered DCT/P407/P188/Tween $80 / \mathrm{NaTC}(0.25 \% / 11 \% / 15 \% / 10 \% / 0.1 \%$ by weight, respectively) resulted in significant anti-tumor efficacy. However, the tumor regression rate for the NaTC group was not statistically different from that for nanomicelles without NaTC. Therefore, overall results suggest that thermosensitive nanomicelles could be a potential dosage form for improvement of the bioavailability and chemotherapeutic profile of DCT.

Keywords: anti-cancer efficiency, bioavailability, docetaxel, liquid suppository, rectal delivery, thermosensitive

\section{Introduction}

Docetaxel (DCT), a broad spectrum taxane drug, is considered to be one of the most potent anti-cancer drugs in a clinical setting with proven efficacy against multiple cancers, including breast cancer, head/neck cancer, gastric cancer, ovarian cancer, and hormone-refractory prostate cancers. ${ }^{1-3}$ However, critical drawbacks, including low aqueous solubility ( $<5 \mu \mathrm{g} / \mathrm{mL}$, highly lipophilic nature) and poor oral bioavailability $(\sim 5 \%$, high first pass metabolism and p-glycoprotein efflux transporter/ CYP3A4 enzyme metabolism) have largely limited its therapeutic application. ${ }^{4,5}$ Many formulation approaches such as nanoparticles, polymer conjugates, nanotransporter, solid dispersions, micro/nanoemulsions (self-nanoemulsifying drug delivery systems), liposome, prodrug, and pre-treatment with p-gp inhibitors, have been attempted in an effort to overcome the drug-related problems. ${ }^{6-10}$ However, none of these approaches improved the bioavailability beyond $\sim 15 \%$ and their limitations far outweighed the therapeutic benefits. At the same time, parenteral administration was associated with severe side effects, including febrile neutropenia, fluid retention, and hypersensitivity reactions that caused patient inconvenience. . $^{41}$ 
Rectal administration of the drug in a suitable dosage form remains a viable alternative to oral and intravenous (IV) administration in order to effectively counter the drawbacks and increase the therapeutic profile of DCT. ${ }^{12,13}$ We recently reported enhanced bioavailability and anti-tumor potential of DCT by incorporation of thermosensitive and bioadhesive nanomicelles. Owing to appreciable bioadhesive interaction with rectal mucosa, the liquid suppository substantially increased the therapeutic concentration of the drug in the blood circulation and remarkable tumor regression was observed with no overt signs of drug or carrier related toxicity. ${ }^{14} \mathrm{Con}$ sistently, in order to further improve the chemotherapeutic potential of DCT, we hypothesized that the presence of a penetration enhancer would facilitate the mucosal internalization of DCT-loaded nanomicelles in the rectum. ${ }^{15}$

Bile salts such as sodium taurocholate (NaTC) have been reported to augment drug permeation across mucosal membranes. In earlier studies, various therapeutic species, including thiocolchicoside, insulin, and alpha-interferon showed enhanced membrane permeability in the presence of NaTC. ${ }^{15,16}$ Although the exact mechanism through which bile salts enhance the absorption and permeability remains elusive, a multitude of pathways, including its ability to increase membrane fluidity, modification of intracellular space, mucous membrane solubilization, change in protein levels on mucosal surface, and reduction in the viscosity of the mucous layer could be proposed. ${ }^{15-17}$ In addition, NaTC embedded in nanomicelles may exhibit a membrane destabilizing effect upon interaction with the rectal mucosa that will facilitate internalization of particles. ${ }^{18}$ Thus, little is known about the influence of NaTC in rectal absorption of chemotherapeutic drugs.

In this study, we set forth to explore the potential of $\mathrm{NaTC}$ for improvement of the bioavailability and anti-tumor efficacy of DCT. Toward this goal, poloxamer, a copolymer of poly(polyethylene)-poly(oxypropylene)-poly(oxyethylene) was selected for the formulation of unique thermosensitive and bioadhesive DCT-loaded nanomicelles. Due to its reverse gelation property, polaxamer remains in liquid state at room temperature $\left(\sim 25^{\circ} \mathrm{C}\right)$, while gelifying at physiological body temperature $\left(\sim 37^{\circ} \mathrm{C}\right) .{ }^{12,14} \mathrm{We}$ designed two delivery systems; DCT/P407/P188/Tween 80 (0.25\%/11\%/15\%/10\% by weight, respectively) and DCT/P407/P188/Tween $80 /$ $\operatorname{NaTC}(0.25 \% / 11 \% / 15 \% / 10 \% / 0.1 \%$ by weight, respectively) . Viscoelastic parameters were studied in terms of gel strength, gelation time, gelation temperature, and mucoadhesive force. Pharmacokinetic study and in vivo anti-tumor studies were performed for investigation of the biological performance of the individual delivery system.

\section{Materials and methods Materials}

DCT was purchased from Taihua Co. (Xi'an, People's Republic of China). Poloxamer 407 (P407) and poloxamer 188 (P188) were procured from BASF (Ludwigshafen, Germany). Tween 80 (polysorbate 80 ) was obtained from DC Chemicals (Seoul, South Korea). All other chemicals were of reagent grade unless otherwise mentioned.

\section{Preparation of DCT-loaded nanomicelles}

DCT-loaded nanomicelles were prepared as previously reported. ${ }^{14}$ Briefly, the surfactant phase was prepared by precisely dissolving $0.25 \mathrm{~g}$ of DCT into $10 \mathrm{~g}$ of Tween 80 . Similarly, the aqueous phase was prepared by dissolving a weighed quantity of P188 (15 g) and P407 (11 g) in ultrapure water maintained at $4^{\circ} \mathrm{C}$. Then, the surfactant phase was gently poured into the poloxamer solution with vigorous agitation. The whole viscous solution was kept at $4^{\circ} \mathrm{C}$ until it became transparent.

\section{Dynamic light scattering measurements}

The hydrodynamic size $(\mathrm{nm})$ and polydispersity index of DCTloaded nanomicelles were measured using Zetasizer Nano ZS (Malvern Instruments, Malvern, UK). The parameters were determined by dynamic light scattering principle at a fixed angle of $90^{\circ}$ at room temperature $\sim 25^{\circ} \mathrm{C}$. Manufacturer provided NanoDTS software (version 6.34; Malvern Instruments, Malvern, UK) was employed for analysis of the results.

\section{Transmission electron microscopy}

The structure and morphology of nanomicelles was determined using a transmission electron microscope (TEM; Hitachi $\mathrm{H}-7600$, Tokyo, Japan) at a voltage of $100 \mathrm{kV}$. Briefly, the final formulation was suitably diluted with distilled water and a drop of micellar preparations was instilled on the carbon-coated copper grid. The particles were allowed to settle for a few minutes and immediately wiped out using soft paper. A solution of $2 \%$ phosphotungstic acid (1:20 ratio of formulation) was then added for negative staining and the particles were then dried.

\section{Viscoelastic property}

\section{Gelation temperature}

Gelation temperature was measured as previously reported. ${ }^{12}$ In brief, a weighed quantity of liquid suppository was placed in a glass vial which was in turn placed in an automated water bath containing a magnetic stirrer and a digital thermometer. A magnetic bar was allowed to rotate $(\sim 100 \mathrm{rpm})$ in the liquid suppository and the temperature was gradually increased from 
$20^{\circ} \mathrm{C}$ to $40^{\circ} \mathrm{C}$ at a rate of $1{ }^{\circ} \mathrm{C}$. With the increase in temperature, the magnetic bar rotation decreased and the point where the rotation stopped was noted as gelation temperature.

\section{Gelation time and gel strength}

The viscosity of liquid suppository under physiological conditions is interpreted in terms of gel strength. The viscosity and gel strength of DCT-loaded nanomicelles were measured using a modern rheometer (Physica MCR 301; Anton Paar, Graz, Austria). The experiments were performed at two different temperature conditions: $25^{\circ} \mathrm{C}$ and $37^{\circ} \mathrm{C}$. The temperature was controlled using a circulating water bath (TC10; Dagan Corporation, Minneapolis, MN, USA) and rheological properties were calculated with the software provided by the manufacturer. The whole set-up was established as reported previously. ${ }^{12}$

\section{Mucoadhesive force}

A healthy female New Zealand rabbit was sacrificed and a tissue section was collected from the fundus part of the rabbit's rectum. Mucoadhesive force was measured as previously reported. Briefly, the tissue section was placed in a transparent glass vial and stored at $37^{\circ} \mathrm{C}$ for 10 minutes. One end of the tissue in the vial was connected to a modified balance while the other end was connected to the vial containing liquid suppository in an adjacent pan. Then, the weight at the other end was slowly increased until both vials were detached from each other. The force required to detach both vials was calculated in terms of detachment force (dyne $/ \mathrm{cm}^{2}$ ) or mucoadhesive force.

\section{Pharmacokinetic study}

\section{Animals}

Male Sprague-Dawley rats (280 \pm 20 g), 6-8 weeks old, were obtained from Nara Biotech (Seoul, South Korea). The rats were housed under ambient conditions $\left(\sim 24^{\circ} \mathrm{C}\right.$ and $50 \%-60 \%$ relative humidity) with 12 hours light-dark cycle. All animal care and handling was performed according to the established principles. The design and protocols for the in vivo study were approved by the Institute of Laboratory Animal Resources, Hanyang University, South Korea.

\section{Administration and blood collection}

The rats were divided into three groups and fasted for 24 hours with free access to water prior to the commencement of experiments. The rats were anesthetized (mixture of Zoletil ${ }^{\circledR}$ [Virbac, Carros, France] and Rompun ${ }^{\circledR}$ [Bayer Animal Health, Monheim, Germany]) and the femoral artery of each rat was catheterized using a polyethylene tube (SP45; Natsume Seisakusho Co., Ltd., Tokyo, Japan) containing heparin solution (100 IU/mL). Rats in the first group received rectal administration of DCT-loaded nanomicelles DCT/ P407/P188/Tween $80(0.25 \% / 11 \% / 15 \% / 10 \%)$ at a dose of $1.11 \mathrm{~mL} / \mathrm{kg}(5 \mathrm{mg} / \mathrm{kg}$ as DCT). Rats in the second group received DCT-loaded nanomicelles DCT/P407/P188/Tween $80 / \mathrm{NaTC}(0.25 \% / 11 \% / 15 \% / 10 \% / 0.1 \%)$ at the same dose of $5 \mathrm{mg} / \mathrm{kg}$ via rectal route. Samples were administered into the rectum of each rat at $4 \mathrm{~cm}$ above the anus with a $45^{\circ}$ slope using a stomach sonde needle. For comparison, rats in the third group received DCT solution via IV route at the same dose as that of previous groups. Injectable DCT solution was prepared by diluting the commercial injectable product (Taxotere $^{\circledR}$; Sanofi-Aventis, Bridgewater, NJ, USA) with 13\% ethanol in water, as recommended by the manufacturer for use in humans, and $300 \mu \mathrm{L}$ of DCT solution $(5 \mathrm{mg} / \mathrm{kg}$ ) was administered to animals. At a specified time point, $0.3 \mathrm{~mL}$ of blood was collected from the femoral artery and plasma was immediately separated by centrifuging the blood samples at $3,000 \mathrm{~g} / 10 \mathrm{~min}$. The samples were stored at $-80^{\circ} \mathrm{C}$ until further analysis.

\section{Blood sample analysis}

One hundred-and-fifty $\mu \mathrm{L}$ of plasma was mixed with $50 \mu \mathrm{L}$ of paclitaxel $(10 \mu \mathrm{g} / \mathrm{mL}$ in acetonitrile $)$ internal standard solution. This plasma mixture was added to $1 \mathrm{~mL}$ of acetonitrile for precipitation of the proteins. After quick vortex-mixing, the mixture was centrifuged at 3,000 $\mathrm{g}$ for 10 minutes, and the supernatant was separated, and evaporated using a high-speed vacuum concentrator (Scan Speed 40; LaboGene Aps, Lynge, Denmark) at $50^{\circ} \mathrm{C}$. The resultant residue was reconstituted with $100 \mu \mathrm{L}$ of the mobile phase and $20 \mu \mathrm{L}$ was injected into the HPLC column. The HPLC system (Agilent 1260 Infinity; Agilent Technologies, Santa Clara, CA, USA) was equipped with an Inertsil ODS-4 column (GL Sciences Inc., Tokyo, Japan; $5 \mu \mathrm{m}, 4.6 \mathrm{~mm}$ ID $\times 250 \mathrm{~mm}$ ) and ultraviolet-visible spectroscopy detector. ACN:0.01M phosphate buffer ( $\mathrm{pH} 5$ ) at a volume ratio of 49/51 was used as a mobile phase. The flow rate was maintained at $1.0 \mathrm{~mL} / \mathrm{min}$ and the detector wavelength was fixed at $230 \mathrm{~nm}$. All pharmacokinetic parameters including maximum plasma concentration $\left(\mathrm{C}_{\max }\right)$, time to reach maximum plasma concentration $\left(\mathrm{T}_{\max }\right)$, area under the whole blood concentration-time curve (AUC), elimination rate constant $\left(\mathrm{K}_{\mathrm{el}}\right)$, and half-life $\left(\mathrm{t}_{1 / 2}\right)$ were estimated using the WinNonlin ${ }^{\mathrm{TM}}$ (Pharsight Corp., Mountain view, CA, USA) program. Values are reported as mean \pm standard deviation $(\mathrm{SD})$ and the data were considered statistically significant at $P<0.05$. 


\section{In vivo anti-tumor study}

A tumor xenograft mouse model was prepared from 7 week old female BALB/c nude mice. ${ }^{14}$ The mice were housed under ambient conditions of 12 hours light-dark cycle according to the animal house regulations $\left(\sim 24^{\circ} \mathrm{C}\right.$ and $\sim 60 \%$ relative humidity). The rats were caged in clean metabolic cages (Tecniplast, Varese, Italy) under sterile, filtered, pathogenfree air, with food and water available ad libitum. The experimental protocols for the animal study were approved by the Animal Care and Use Committee of the College of Pharmacy, Yeungnam University. One $\times 10^{6}$ SCC-7 cells in $100 \mu \mathrm{L}$ phosphate-buffered saline was subcutaneously injected into the right flank of each mouse and continuously monitored for tumor development. The experiment was started approximately 10 days after cell injection when the tumor volume reached $\sim 150 \mathrm{~mm}^{3}$. The mice were divided into three groups, with seven mice in each group. Mice in the first group received rectal administration of DCT-loaded nanomicelles DCT/P407/P188/Tween $80(0.25 \% / 11 \% / 15 \% / 10 \%)$ at a dose of $5 \mathrm{mg} / \mathrm{kg}$ DCT. Mice in the second group received DCT-loaded nanomicelles DCT/P407/P188/Tween 80/NaTC $(0.25 \% / 11 \% / 15 \% / 10 \% / 0.1 \%)$ at the same dose of $5 \mathrm{mg} / \mathrm{kg}$ via rectal route. The drug treatment was administered once every 3 days, at days 1, 4, 7, and 10. The length and width of the tumor in each mouse was measured using calipers. The antitumor effect of the DCT-loaded nanomicelles was compared to the nanomicelles group containing NaTC by observing tumor volume reduction and measuring total body weight.
The anti-tumor efficacy was calculated based on the tumor volume measurement using the equation $\mathrm{V}=0.5 \times$ longest diameter $\times$ shortest diameter. After the study period, mice were sacrificed according to the ethical guidelines. ${ }^{19}$ Results are expressed as mean tumor volume (control, C/test, T) against time. Criteria for anticancer activity were $\mathrm{T} / \mathrm{C} \%<60 \%$.

\section{Statistical analysis}

All data were expressed as mean \pm SD. Data were statistically analyzed by ANOVA with Student-Newman-Keuls post-hoc test. A $P$-value of less than 0.05 was considered statistically significant.

\section{Results \\ Physicochemical characterization of DCT-loaded nanomicelles}

DCT-loaded nanomicelles were successfully formulated with $0.25 \%$ DCT, $15 \%$ P $188,11 \%$ P $407,10 \%$ Tween 80 , and $0.1 \%$ NaTC (Figure 1). Recently, various process and formulation related variables were optimized in order to adjust the thermogelling and mucoadhesive properties for effective rectal administration (Table 1). ${ }^{12}$ The rate and extent of nanoparticle internalization is highly dependent on the particle size and size distribution of DCT-loaded nanomicelles. In this regard, results of dynamic light scattering analysis showed that micelles were nanosized with an average size around $\sim 15 \mathrm{~nm}$ with a narrow poly dispersity index (0.2) (Figure 2A). The particle size almost remained the same for the inclusion of NaTC as a<smiles>C=C(COCCOCCO)OCCO</smiles>

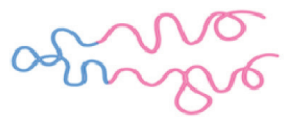

Poloxamer
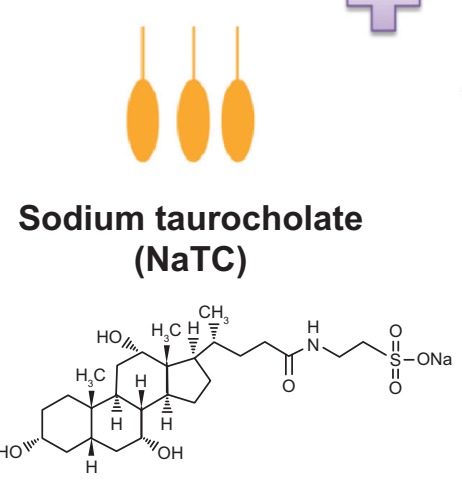
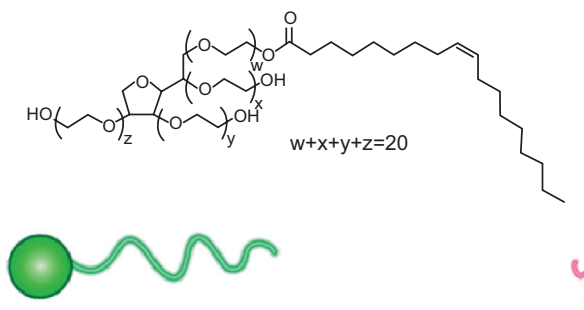

Tween 80

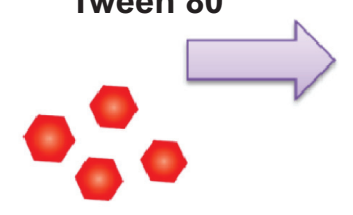

Docetaxel (DCT)

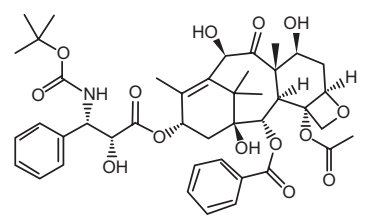

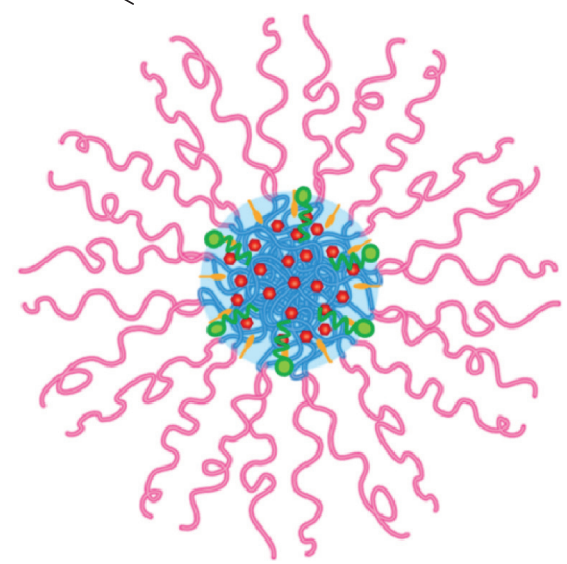

DCT-loaded nanomicelles

Figure I Schematic illustration of the preparation of DCT-loaded nanomicelles containing $\mathrm{NaTC}$. 
permeation enhancer. This might be attributed to the very low concentration of NaTC employed for formulation of nanosized micelles. TEM images further confirmed the size and shape of the NaTC containing nanomicelles in the dried state. The particles were distinctly spherical with well-maintained perfect boundaries and presented a uniform distribution of particles. Lack of any clusters and conglomerates suggests good particle integrity and physical stability. The micellar appearance of particles was clearly visible in the magnified image.

A gelation temperature range between $33^{\circ} \mathrm{C}-37^{\circ} \mathrm{C}$ is an important prerequisite of the liquid suppository intended for administration in the rectal cavity. ${ }^{19}$ It is the temperature at which phase transition occurs between liquid and gel state (Figure 2B). In general, a lower gelation temperature $\left(<30^{\circ} \mathrm{C}\right)$ would cause difficulty in handling and administration, while a higher gelation temperature $\left(<36^{\circ} \mathrm{C}\right)$ would result in a leakage and drug absorption problem..$^{20}$ In the current study, gelation temperature was at the
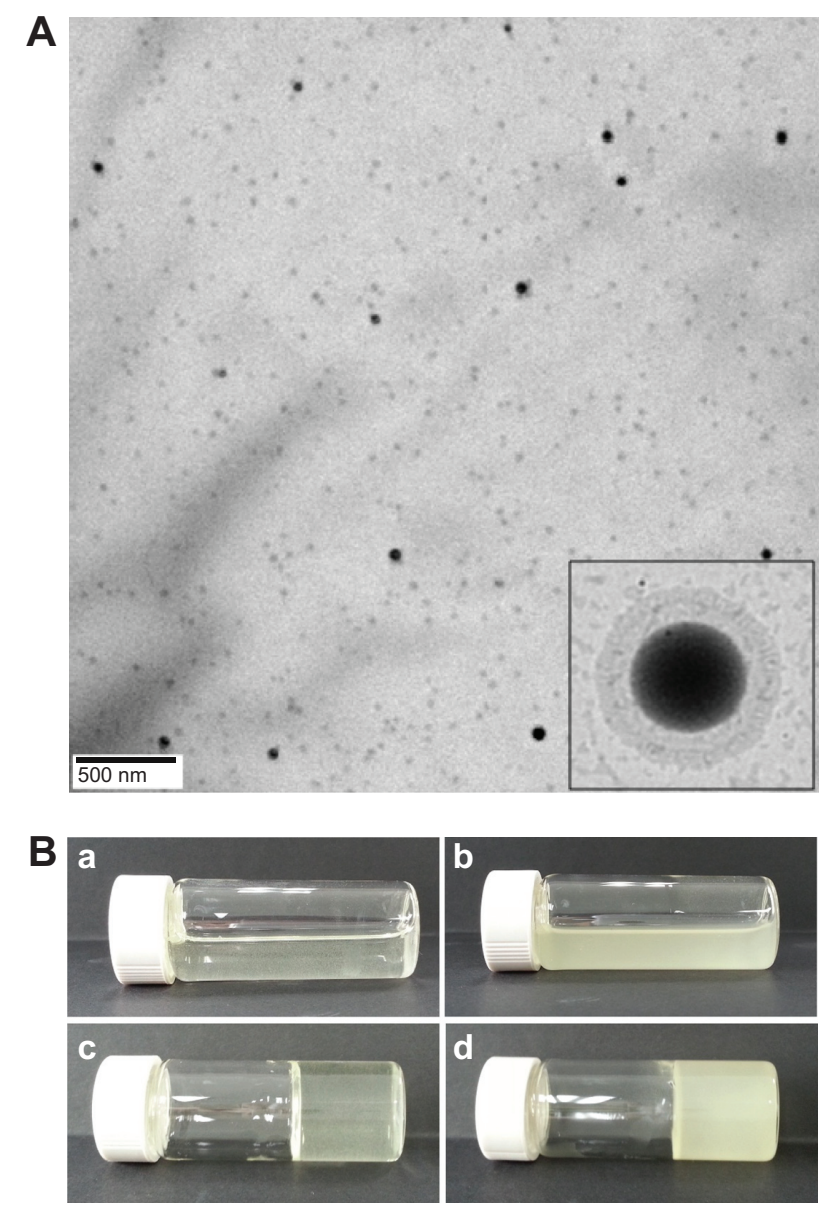

Figure 2 (A) TEM image of DCT-loaded nanomicelles containing NaTC. (B) Optical images of DCT-loaded nanomicelles at room temperature (liquid; a,b) and biological temperature (solid; c,d). Images correspond to nanomicelles without $(a, c)$ and with $\mathrm{NaTC}(\mathrm{b}, \mathrm{d})$.

Abbreviations: TEM, transmission electron microscope; DCT, docetaxel; NaTC, sodium taurocholate. optimized range $\left(\sim 33^{\circ} \mathrm{C}\right)$, sufficient to handle and release its content in the rectum. Previously, we have optimized the appropriate concentration of P407 and P188 such that the thermosensitive micelles formed a gel below the physiological body temperature. ${ }^{12}$ The addition of NaTC caused a slight increase in the gelation temperature.

Viscosity of the formulation is another important factor in development of DCT-loaded thermosensitive nanomicelles. In general, poloxamer-based nanomicelles exhibit a viscosity threshold of $40 \mathrm{~Pa} \cdot \mathrm{s}$ at $36.5^{\circ} \mathrm{C}$. However, the addition of other ingredients might influence the rheological property of formulations. ${ }^{21}$ In the current study, the viscosity was around $\sim 25 \mathrm{~Pa} \cdot \mathrm{s}$, which was in the region of threshold viscosity, indicating that the formulation holds appreciable gel strength at physiological conditions (Figure 3). The viscosity was unaltered for the addition of NaTC to DCT-loaded nanomicelles, which was between 160-190 mPa.s for both formulations at $25^{\circ} \mathrm{C}$. Such low viscosity at room temperature would enable easy administration of formulations in the rectal cavity. ${ }^{22}$ Syringe-ability requires a threshold viscosity below which it is easy to administer, while at the upper end the sonde and syringe would eventually break. For successful administration of DCT, the nanomicelles should possess quicker gelation time with high gel strength. A threshold gel strength is required in order to allow the suppository to stay in contact with the rectal mucosa, whereas below this threshold it, will flow like liquid. The time required to reach

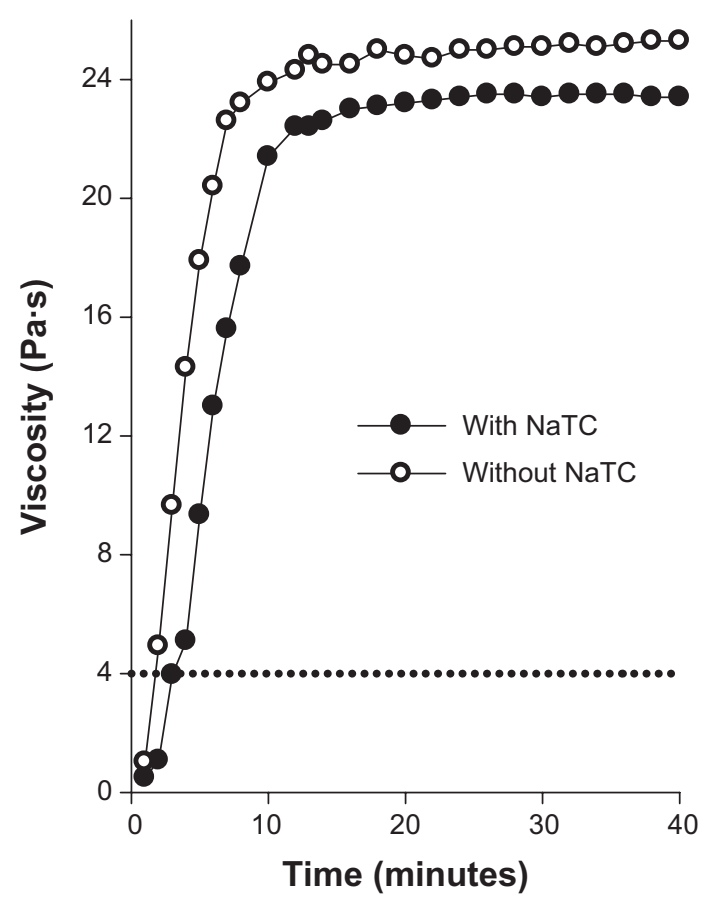

Figure 3 Rheological behavior of DCT-loaded nanomicelles for rectal administration at $36.5^{\circ} \mathrm{C}$.

Abbreviations: DCT, docetaxel; $\mathrm{NaTC}$, sodium taurocholate. 
this threshold level $\left(\sim 4,000 \mathrm{mPa} \cdot \mathrm{s}\right.$ at $\left.36^{\circ} \mathrm{C}\right)$ will be noted as gelation time. In this study, we observed a gel strength around $\sim 25,000$ ( $\mathrm{mPa} \cdot \mathrm{s})$ with a short gelation time of 2 minutes. Although the gelation time showed a slight increase with the addition of NaTC, it was less than 3 minutes, satisfying the criteria for an effective thermosensitive gel. ${ }^{12}$

In order to attain maximum bioavailability and chemotherapeutic response of DCT, mucoadhesive force of thermosensitive nanomicelles to interact with the rectal mucosa is of great importance. ${ }^{19,21}$ Both nanomicelles exhibited appreciable mucoadhesive force $\left(\sim 18\right.$ dyne $\left./ \mathrm{cm}^{2}\right)$ to be retained in the upper part of the rectum. Generally, a stronger interaction with the mucosa can prevent the formulations from reaching the anus, and, at the same time, longer duration of contact will eventually increase the rate and extent of drug absorption. The poloxamers (P407 and P188) exhibited bioadhesive properties because their oxyethylene and oxypropylene functional groups form hydrogen bonds with the sialic acid of oligosaccharide chains on the rectal mucous membranes..$^{21,23}$

\section{In vivo pharmacokinetic study}

For comparison of the bioavailability profiles of DCT-loaded nanomicelles with, and without NaTC, pharmacokinetic studies were performed on rats. The pharmacokinetic behavior of nanomicelles was compared with that of IV DCT solution. For the commercial injectable product, Taxotere ${ }^{\circledR}$, was diluted with $13 \%$ ethanol in water before injection. IV DCT solution was used as a control for comparison of the pharmacological performance after rectal and parenteral administration, respectively. The plasma concentration-time profiles of DCT (as DCT solution and DCT-loaded nanomicelles) following IV and rectal administration at a dose of $5 \mathrm{mg} / \mathrm{kg}$ are shown in Figure 4. The total dose of DCT was kept lower in order to avoid drug-related adverse effects that might influence the nature of the experiments. Following administration of commercially injectable DCT solution, the plasma concentration showed a linear decrease from $\sim 10,000 \mathrm{ng} / \mathrm{mL}$ to $<50 \mathrm{ng} / \mathrm{mL}$ within the first 4 hours of the study period. During the same time, $>500 \mathrm{ng} / \mathrm{mL}(P<0.01)$ was maintained in the groups rectally administered with DCT-loaded nanomicelles. Consistent with our hypothesis, DCT-loaded nanomicelles containing NaTC showed significantly higher plasma concentration at certain time points. Specifically, at the end of 2 hours, nanomicelles containing bile salt showed more than $>800 \mathrm{ng} / \mathrm{mL}$ of DCT $(P<0.05)$, compared to $\sim 600 \mathrm{ng} / \mathrm{mL}$ from nanomicelles without NaTC. The same trend continued up to 3 hours, after which the plasma levels were similar for the remainder of the study period.

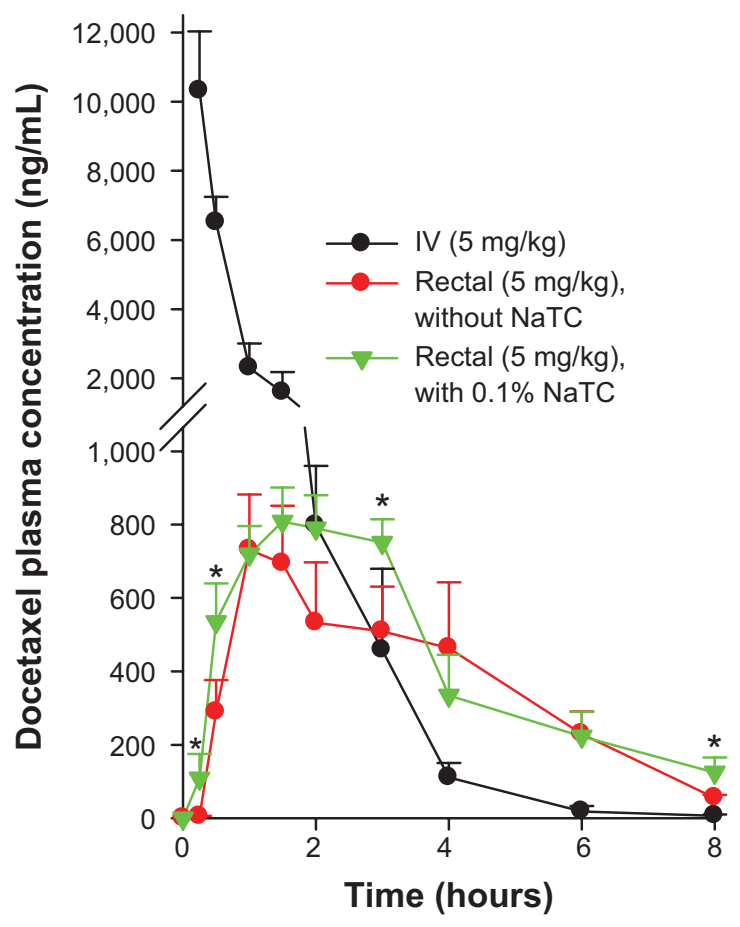

Figure 4 Plasma concentration-time profiles of DCT-loaded nanomicelles with and without $\mathrm{NaTC}$ in rats after rectal administration.

Notes: The DCT-loaded nanomicelle with NaTC was composed of DCT/P407/ PI88/Tween $80 / \mathrm{NaTC}(0.25 \% / 1 / \% / 15 \% / 10 \% / 0.1 \%)$. IV DCT solution was used as a control and each value represents the mean \pm standard deviation $(n=6)$. *Significantly higher than plain nanomicelle group $(P<0.05)$.

Abbreviations: DCT, docetaxel; NaTC, sodium taurocholate; IV, intravenous; $\mathrm{Na}$, sodium.

A list of the corresponding pharmacokinetic parameters is shown in Table 2. Most importantly, bile salt containing formulations exhibited a very high bioavailability of $32.8 \%$ which, to the best of our knowledge, is the highest reported value. The AUC remained at 3,405.6 and 2,940.75 $\pm 783 \mathrm{h.ng} / \mathrm{mL}$ for micelles with, and without NaTC, respectively. In addition, bile salt relatively improved the $\mathrm{t}_{1 / 2}(\sim 2.5 \mathrm{~h})$ and decreased the elimination rate constant $\mathrm{K}_{\mathrm{el}}\left(0.29 \mathrm{~h}^{-1}\right)$ of DCT in rats. This result is in compliance with our earlier postulation that NaTC could enhance the plasma drug level when administered rectally.

\section{In vivo anti-tumor study}

In order to assess the question of whether inclusion of bile salt resulted in any significant anti-tumor efficacy, DCT-loaded

Table I Rheological properties of DCT-loaded nanomicelles without or with $0.1 \% \mathrm{NaTC}$

\begin{tabular}{lll}
\hline Properties & Without NaTC & With NaTC \\
\hline Gelation temperature $\left({ }^{\circ} \mathrm{C}\right)$ & $33.0 \pm 0.5$ & $34.2 \pm 0.6$ \\
Viscosity at $25^{\circ} \mathrm{C}(\mathrm{mPa} \cdot \mathrm{s})$ & $192.0 \pm 2.0$ & $162.0 \pm 3.0$ \\
Gel strength $\left(\times 10^{2} \mathrm{mPa} \cdot \mathrm{s}\right)$ & $252.0 \pm 1.0$ & $234.0 \pm 1.0$ \\
Gelation time (minutes) & 2.0 & 2.9 \\
Mucoadhesive force $\left(\times 10^{2} \mathrm{dyne} / \mathrm{cm}^{2}\right)$ & $18.5 \pm 1.7$ & $17.3 \pm 2.3$ \\
\hline
\end{tabular}

Note: Each value represents the mean \pm standard deviation $(n=3)$. Abbreviations: DCT, docetaxel; NaTC, sodium taurocholate. 
Table 2 Pharmacokinetic parameters of rectally administered DCT-loaded nanomicelles (without or with $0.1 \% \mathrm{NaTC}$ ) and intravenous DCT solution at a dose of $5 \mathrm{mg} / \mathrm{kg}$

\begin{tabular}{llll}
\hline Parameters & Rectal, without NaTC & Rectal, with 0.1\% NaTC & Intravenous \\
\hline AUC $(\mathrm{h} \cdot \mathrm{ng} / \mathrm{mL})$ & $2,940.8 \pm 783.5$ & $3,405.8 \pm 6 \mid 14.7$ & $10,393.0 \pm 1377.2$ \\
$\mathrm{~T}_{\text {max }}(\mathrm{h})$ & $1.17 \pm 0.29$ & $1.67 \pm 0.29$ & - \\
$\mathrm{C}_{\text {max }}(\mathrm{ng} / \mathrm{mL})$ & $739.5 \pm 143.2$ & $808.2 \pm 92.9$ & $10,316.3 \pm 1717.6$ \\
$\mathrm{~T}_{1 / 2}(\mathrm{~h})$ & $1.49 \pm 0.46$ & $2.47 \pm 0.58$ & $0.89 \pm 0.17$ \\
$\mathrm{~K}_{\mathrm{el}}\left(\mathrm{h}^{-1}\right)$ & $0.50 \pm 0.15$ & $0.29 \pm 0.08$ & $0.80 \pm 0.15$ \\
Absolute bioavailability $(\%)$ & 28.3 & 32.8 & - \\
\hline
\end{tabular}

Note: Each value represents the mean \pm standard deviation $(n=6)$.

Abbreviations: DCT, docetaxel; NaTC, sodium taurocholate; AUC, area under the whole blood concentration-time curve; h, hour; $\mathrm{T}_{\text {max }}$, time to reach maximum plasma concentration; $\mathrm{C}_{\text {max }}$, maximum plasma concentration; $\mathrm{T}_{1 / 2}$, half life; $\mathrm{K}_{\mathrm{el}}$, elimination rate constant.

nanomicelles were administered rectally in SCC-7 tumorbearing nude mice. For this reason, respective formulations were given four times every 3 days and the anti-tumor efficacy was interpreted from the reduction in the tumor volume. As shown in Figure 5, tumor size increased proportionately at every time point and rapidly attained maximum tumor volume $\left(-3,200 \mathrm{~mm}^{3}\right)$ for control at day 17 . On the other hand, both DCT-loaded nanomicelles significantly reduced the tumor growth of xenograft mice $(P<0.001)$. Compared to control, nanomicelle groups showed marked inhibition of tumor progression. Although we observed relatively higher pharmacokinetic parameters for NaTC containing nanomicelles, it did not result in any significant improvement in the anti-tumor efficacy of DCT. These results suggest that rectal

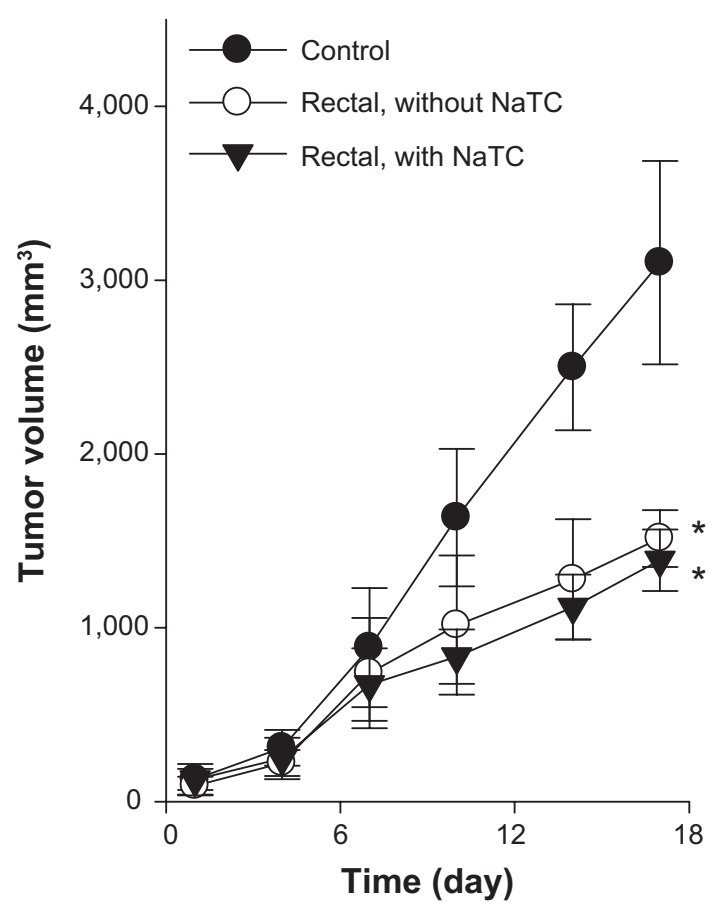

Figure 5 Anti-tumor efficacy of DCT-loaded nanomicelles with and without NaTC after rectal administration.

Notes: Each value represents the mean \pm standard deviation $(n=7)$. *Significantly smaller than control group $(P<0.01)$.

Abbreviations: DCT, docetaxel; $\mathrm{NaTC}$, sodium taurocholate. administration of nanomicelles was very effective in controlling tumor development, even if the inclusion of bile salt had no statistically significant effect on the overall efficacy. In addition, none of the formulations resulted in any decrease in body weight, indicating that the DCT-loaded nanomicelles were quite safe (Figure 6).

\section{Discussion}

DCT, a potent chemotherapeutic agent, is effective against a wide spectrum of tumor malignancies. ${ }^{24}$ However, its low gastrointestinal fluid solubility, poor bioavailability, high hepatic first pass metabolism, and p-gp mediated efflux transport make it a poor choice of drug for oral delivery. ${ }^{5}$ At the same time, the injectable formulation of DCT (eg, Taxotere ${ }^{\circledR}$ and Duopafei ${ }^{\circledR}$

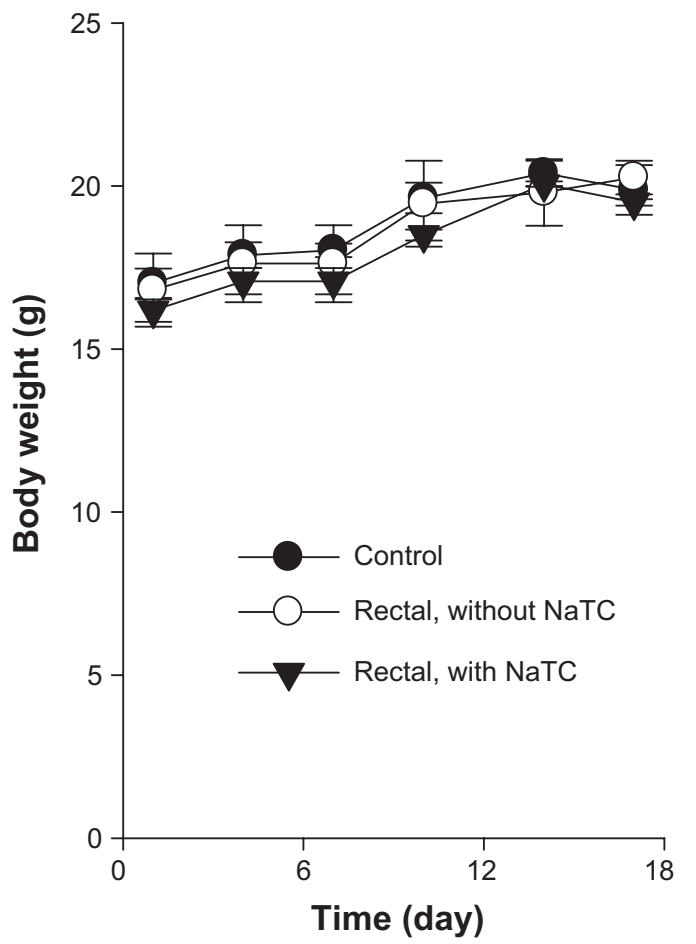

Figure 6 Body weight changes of tumor-bearing nude mice after rectal administration of DCT-loaded nanomicelles with and without NaTC.

Note: Each value represents the mean \pm standard deviation $(n=7)$.

Abbreviations: DCT, docetaxel; NaTC, sodium taurocholate. 
[Qilu Pharma Co., Ltd., Jinan, China]) caused unwanted side effects, including neurotoxicity, neutropenia, fluid retention, and hypersensitivity reactions, which may limit its systemic application. ${ }^{11}$ Under these circumstances, where administration through other popular routes is undesirable, rectal administration offers an alternative route of delivery. ${ }^{12}$ The rectal route offers unique advantages, such as immediate proximity to systemic circulation, avoidance of first pass metabolism, and presence of only a few p-gp membrane transporters that would improve the plasma drug level. ${ }^{25,26} \mathrm{We}$ recently demonstrated that drug-loaded thermosensitive nanomicelles capable of transforming from liquid phase to gel phase at physiological body conditions would be quite suitable. ${ }^{14}$ In addition, a relatively faster absorption of the drug with improved plasma profile for respective drugs was shown without causing any discomfort to the animal. However, in the current study, the potential of bile salt to improve the bioavailability and chemotherapeutic response of DCT was investigated. We postulated that in the presence of NaTC, the permeability of DCT across the rectal mucosa would increase.

Taking all of these facts into consideration, we designed two delivery systems, one with bile salt and one without bile salt; DCT/P407/P188/Tween $80(0.25 \% / 11 \% / 15 \% / 10 \%)$ and DCT/ P407/P188/Tween 80/NaTC (0.25\%/11\%/15\%/10\%/0.1\%). Poloxamer mixtures P407 and P188 were employed as liquid suppository bases that can become gel at the range of physiological temperature. In addition, poloxamer groups possess favorable characteristics, including excellent biocompatibility, good water solubility, high solubilizing capacity, and negligible levels of skin irritation. ${ }^{12,27}$ Tween 80 acts as an additional solubilizing agent, and can form a eutectic mixture with DCT. As reported previously, a eutectic mixture has greater solubility than free drug. Specifically, addition of bile salt was believed to increase the rate and extent of DCT absorption from the rectal mucosa. ${ }^{28}$ Furthermore, bile salt facilitates the internalization of nanomicelles via multiple mechanisms such as membrane-destabilizing effect and membrane fluidity.

The DCT-loaded nanomicelles exhibited a nanosize of approximately $\sim 15 \mathrm{~nm}$ with a perfect spherical shaped particle. The addition of NaTC did not influence the size of the particle owing to a relatively low concentration in the mixture. Both nanomicelles maintained optimum characteristics required to exhibit effective thermo-reversible property. Handling and administration of the formulated nanomicelles was easy due to their low viscosity at room temperature, and they rapidly gelled in the body due to short gelation time and high gel strength, and possessed sufficient mucoadhesive force to hold a gelled suppository within the rectal region for a prolonged period of time.
The primary aim of this study was to investigate the role of bile salt in improving the bioavailability level and therapeutic response of the drug in the blood circulation. For this reason, both nanomicelles were administered rectally at an equivalent dose of $5 \mathrm{mg} / \mathrm{kg}$ of DCT. The results indicate that nanomicelles containing NaTC significantly increased the plasma concentration of DCT until 4 hours $(P<0.01)$, after which the plasma levels were similar for both formulations. Specifically, during the first 30 minutes, the DCT from the bile salt group was 25 -fold higher than that of the other group with no bile salt. This initial higher concentration might be attributed to the high permeation of DCT across rectal mucosa due to the presence of bile salt. However, it can be expected that once in the blood circulation, the drug will be removed at the same rate, resulting in similar plasma concentration after 4 hours of the study period. The pharmacokinetic parameters also clearly demonstrate the difference between the two formulations, with, and without bile salt. The bile salt relatively improved the bioavailability profile of DCT (>30\%) with significantly higher AUC value. Of particular importance, bile salt augmented the $t_{1 / 2}$ of the drug by 1.7 -fold in comparison with the one with no permeation enhancer and reduced the elimination constant by half. As expected, NaTC enhanced the absorption and bioavailability of DCT in the blood circulation, even if it was not very marked. These observations can be interpreted in two ways: a) first, a relatively higher concentration of DCT could be attributed to the presence of NaTC in the nanomicelles. NaTC is known to increase membrane fluidity, solubilize the mucous membrane, interact with the intracellular space, increase water flux, reduce the viscosity of the mucous layer, and destabilize the mucous membrane $\mathrm{e}^{15}$ (all of these processes help transport the drug across the mucosa, resulting in an initial increase in plasma levels); b) in the second phase of the plasma concentration-time graph, the influence of bile salt was diminished and the concentration from both formulations was similar. This could be attributed to many reasons, including rapid clearance of DCT from the blood circulation, dependence on concentration gradient, or the merging of bile salts that may cause formation of swollen mixed micelles that will eventually form an aggregate impeding the mobility of particles toward the mucosa. Although one can expect prolonged contact between nanomicelles and mucosal membranes, the mutual interactions weaken due to the mobility of the subject, which may hamper the performance of bile salt in the endothelium. It has already been reported that bile salt acts as a shuttle/cover to transport the particles and this process is only possible when the suppository lies in close contact with the rectal mucosa. ${ }^{16,17}$ Nevertheless, both rectal 
formulations exhibited a high absolute bioavailability of more than $28 \%$, which could be attributed to a combination of various factors including excellent appreciable gel strength, high mucoadhesive force, and nanosized spherical particles that would facilitate the internalization. ${ }^{13,14}$ This bioavailability is nearly 10 -fold higher than that of the orally administered DCT, as we reported previously. ${ }^{29}$ In the same study, we reported a $17 \%$ increase in bioavailability of the drug by DCT-loaded self-nanoemulsifying drug delivery systems (oral), which was the highest reported value until this point in time. By rectal administration of nanomicelles with bile salt, we almost doubled the availability of the drug in the systemic circulation ( $\sim 33 \%$ bioavailability). This significant bioavailability result indisputably vouches for the distinct enhancement in the anti-tumor drug response.

Having investigated the influence of bile salt on the bioavailability level, we next evaluated the question of whether or not the same response is replicated in the chemotherapeutic potential of DCT. Tumor fragments derived from SCC-7 cells were implanted subcutaneously into xenograft nude mice. The tumor bearing mice were treated with respective formulations at a dose of $5 \mathrm{mg} / \mathrm{kg}$ when the tumor size reached the pre-determined volume $\left(\sim 150 \mathrm{~mm}^{3}\right)$. As can be seen in Figure 5, both formulations delayed tumor growth significantly by comparison with those of the control group $(P<0.01)$. Although DCT from both micellar formulations showed marked tumor inhibition, the anti-cancer efficacy was indistinguishable among the two groups. A tumor regression of $50 \%$ and $55 \%$ in xenograft mice can be well correlated with the plasma drug level and pharmacokinetic parameters. In general, anti-cancer efficacy of one system will differ from that of another if there is a significant difference in plasma circulation time and $t_{1 / 2}$ parameters, as a prolonged blood circulation time will increase the chance of drug internalization into cancer cells via the enhanced permeability and retention (EPR) effect. ${ }^{30}$ However, in the current study, after administration for 4 hours, the plasma levels of DCT were similar for both groups. Although significant differences in pharmacokinetic parameters were observed during the initial time points, it was not sufficient to elicit a pronounced tumor response.

Safety profiles of DCT formulations were investigated in terms of changes in the average body weight (Figure 6). A decrease in body weight is regarded as an index for drugrelated organ toxicity. In the current study, neither acute adverse effects nor death by toxicity was observed from either formulation, ensuring its complete safety. This can be best explained by the slow uptake of particles from the rectum and low plasma concentration of drugs, which might be too low to produce any toxic effects. Some studies have reported that mice treated with commercial injection shed at least $10 \%$ of their body weight and were found to be lethargic with overt signs of severe toxicity. ${ }^{14,30-32}$ In contrast, orally administered DCT solution did not have any effect on tumor progression, and tumor volume increased rapidly, equal to that of control. ${ }^{14}$ The poor response of oral DCT solution was consistent with the poor bioavailability profile $(\sim 2 \%)$, high first pass metabolism, and rapid plasma clearance, where only a small amount of drug remained in the circulation to elicit its action. All of these observations led to the speculation that Taxotere ${ }^{\circledR}$ was very toxic, while the orally administered drug solution did not have any effect on tumor response, and therefore, both are unsuitable. ${ }^{30}$ On the other hand, DCTloaded nanomicelles with, and without bile salt were very efficacious in controlling the tumor burden, and, at the same time, completely safe. Furthermore, enhanced anti-tumor efficacy of DCT-loaded nanomicelles was partly attributed to the ability to counter p-gp (ABCB1) mediated efflux activity. It has been reported that pluronic (188 and 407) as well as Tween 80 may inhibit the function of $\mathrm{p}$-gp by changing the membrane-fluidity. Specifically, it binds to the hydrophobic domain of the p-gp that may change its secondary and/or tertiary structure and reduce its function. Therefore, modulation of p-gp might increase the intracellular accumulations of DCT while decreasing the drug efflux toward the extracellular cells leading to augmented therapeutic efficacy.

\section{Conclusion}

A thermosensitive and mucoadhesive DCT-loaded nanomicelle DCT/P407/P188/Tween 80/NaTC $(0.25 \% / 11 \% / 15 \% / 10 \% / 0.1 \%)$ was successfully formulated for investigation of the influence of bile salt in bioavailability enhancement and tumor killing potential of DCT, when administered rectally. DCT-loaded nanomicelles for rectal administration possessed sufficient viscoelastic properties to present in the upper part of the rectum for the specified time. The pharmacokinetic results showed that NaTC was influential in improving the half-life and plasma level of DCT. However, the plasma concentration of DCT remained similar from both nanomicelle formulations after 4 hours of the study period. Although the elevated plasma level of DCT from the bile salt group did not enhance the anti-tumor potential, this formulation showed significant anti-tumor efficacy, while avoiding the adverse effects reported with the latter. Therefore, it can be expected that DCT-loaded nanomicelles could reduce the drug-related side effects, including hypersensitivity reactions and fluid retention, while retaining the potential anti-tumor efficacy in clinical subjects. 


\section{Acknowledgment}

This work was supported by the National Research Foundation of Korea (NRF) grant funded by the Korea government (MEST) (NRF-2013M4A1035382 and 2012R1A1A1039059).

\section{Disclosure}

The authors report no conflicts of interest in this work.

\section{References}

1. Zhao P, Astruc D. Docetaxel nanotechnology in anticancer therapy. Chem Med Chem. 2012;7(6):952-972.

2. Kintzel PE, Michaud LB, Lange MK. Docetaxel-associated epiphora. Pharmacotherapy. 2006;26(6):853-867.

3. Sanna V, Roggio AM, Posadino AM, et al. Novel docetaxel-loaded nanoparticles based on poly(lactide-co-caprolactone) and poly(lactideco-glycolide-co-caprolactone) for prostate cancer treatment: formulation, characterization, and cytotoxicity studies. Nanoscale Res Lett. 2011;6(1):260.

4. Hu K, Cao S, Hu F, Feng J. Enhanced oral bioavailability of docetaxel by lecithin nanoparticles: preparation, in vitro, and in vivo evaluation. Int J Nanomedicine. 2012;7:3537-3545.

5. Cho HJ, Yoon HY, Koo H, et al. Self-assembled nanoparticles based on hyaluronic acid-ceramide (HA-CE) and Pluronic ${ }^{\circledR}$ for tumor-targeted delivery of docetaxel. Biomaterials. 2011;32(29):7181-7190.

6. Yan YD, Kim DH, Sung JH, Yong CS, Choi HG. Enhanced oral bioavailability of docetaxel in rats by four consecutive days of pretreatment with curcumin. Int J Pharm. 2010;399(1-2):116-120.

7. Esmaeili F, Dinarvand R, Ghahremani MH, et al. Docetaxel-albumin conjugates: preparation, in vitro evaluation and biodistribution studies. J Pharm Sci. 2009;98(8):2718-2730.

8. Straubinger RM, Balasubramanian SV. Preparation and characterization of taxane-containing liposomes. Methods Enzymol. 2005;391:97-117.

9. Chen J, Qiu L, Hu M, Jin Y, Han J. Preparation, characterization and in vitro evaluation of solid dispersions containing docetaxel. Drug Dev Ind Pharm. 2008;34(6):588-594.

10. Zhang H, Dou J, Zhai Y, Liu A, Zhai G. Advances in the formulations of non-injection administration of docetaxel. J Drug Target. 2014;22(2): 87-94.

11. Baker J, Ajani J, Scotte F, et al. Docetaxel-related side effects and their management. Eur J Oncol Nurs. 2009;13(1):49-59.

12. Yeo WH, Ramasamy T, Kim DW, et al. Docetaxel-loaded thermosensitive liquid suppository: optimization of rheological properties. Arch Pharm Res. 2013;36(12):1480-1486.

13. Yuan Y, Ying C, Li Z, et al. Thermosensitive and mucoadhesive in situ gel based on poloxamer as new carrier for rectal administration of nimesulide. Int J Pharm. 2012;430(1-2):114-119.

14. Seo YG, Kim DW, Yeo WH, et al. Docetaxel-loaded thermosensitive and bioadhesive nanomicelles as a rectal drug delivery system for enhanced chemotherapeutic effect. Pharm Res. 2013;30(7):1860-1870.

15. Yamamoto A, Tatsumi H, Maruyama M, Uchiyama T, Okada N, Fujita T. Modulation of Intestinal Permeability by Nitric Oxide Donors: Implications in Intestinal Delivery of Poorly Absorbable Drugs. J Pharmacol Exp Ther. 2001;296(1):84-90.
16. Van Hasselt PM, Janssens GEPJ, Slot TK, et al. The influence of bile acids on the oral bioavailability of vitamin $\mathrm{K}$ encapsulated in polymeric micelles. J Control Release. 2009;133(2):161-168.

17. Bondesson E, Bengtsson T, Nilsson LE, Wollmer P. Effects of Sodium Taurocholate on the Absorption of Inhaled 99mTc-DTPA. Pharm Res. 2006;23(9):2122-2128.

18. Niua M, Lu Y, Hovgaard L, et al. Hypoglycemic activity and oral bioavailability of insulin-loaded liposomes containing bile salts in rats: the effect of cholate type, particle size and administered dose. Eur $J$ Pharm Biopharm. 2012;81(2):265-272.

19. Jin YJ, Termsarasab U, Ko SH, et al. Hyaluronic acid derivative-based self-assembled nanoparticles for the treatment of melanoma. Pharm Res. 2012;29(12):3443-3454.

20. Choi HG, Kim MH, Lee MK, Kim CK. Effect of additives on the physicochemical properties of liquid suppository. Int J Pharm. 1999;190(1): $13-19$.

21. Barakat NS. In vitro and in vivo characteristics of a thermogelling rectal delivery system of etodolac. APPS Pharm Sci Tech. 2009;10(3): 724-731.

22. Choi HG, Jung JH, Ryu JM, et al. Development of in situ-gelling and mucoadhesive acetaminophen liquid suppository. Int $J$ Pharm. 1998;165(1):33-44.

23. Yong CS, Yang CH, Rhee JD, et al. Enhanced rectal bioavailability of ibuprofen in rats by poloxamer 188 and menthol. Int J Pharm. 2004;269(1):169-176.

24. Yong CS, Choi JS, Quan QZ, et al. Effect of sodium chloride on the gelation temperature, gel strength and bioadhesive force of poloxamer gels containing diclofenac sodium. Int J Pharm. 2001;226(1):195-205.

25. Ray A, Larson N, Pike DB, et al. Comparison of active and passive targeting of docetaxel for prostate cancer therapy by HPMA copolymerRGDfK conjugates. Mol Pharm. 2011;8(4):1090-1099.

26. Sakai M, Hobara N, Hokama N, et al. Increased bioavailability of tacrolimus after rectal administration in rats. Biol Pharm Bull. 2004;27(9): 1480-1482.

27. Elsabahy M, Perron ME, Bertrand N, Yu GE, Leroux JC. Solubilization of docetaxel in poly(ethylene oxide)-block-poly(butylene/styrene oxide) micelles. Biomacromolecules. 2007;8(7):2250-2257.

28. Mayol L, Biondi M, Quaglia F, et al. Injectable thermally responsive mucoadhesive gel for sustained protein delivery. Biomacromolecules. 2011;12(1):28-33.

29. Yang Y, Wang J, Zhang X, Lu W, Zhang Q. A novel mixed micelle gel with thermosensitive property for the local delivery of docetaxel. J Control Release. 2009;135(2):175-182.

30. Seo YG, Kim DH, Ramasamy T, et al. Development of docetaxel-loaded solid self-nanoemulsifying drug delivery system (SNEDDS) for enhanced chemotherapeutic effect. Int J Pharm. 2013;452(1-2):412- 420.

31. Ramasamy T, Kim JH, Choi HG, Yong CS, Kim JO. Novel dual drugloaded block ionomer complex micelles for enhancing the efficacy of chemotherapy treatments. J Biomed Nanotechnol. 2014;10(7): 1304-1312.

32. Zhang Y, Wang X, Wang J, Zhang X, Zhang Q. Octreotide-modified polymeric micelles as potential carriers for targeted docetaxel delivery to somatostatin receptor overexpressing tumor cells. Pharm Res. 2011; 28(5):1167-1178.
International Journal of Nanomedicine

\section{Publish your work in this journal}

The International Journal of Nanomedicine is an international, peerreviewed journal focusing on the application of nanotechnology in diagnostics, therapeutics, and drug delivery systems throughout the biomedical field. This journal is indexed on PubMed Central, MedLine, CAS, SciSearch $\AA$, Current Contents $\AA /$ Clinical Medicine,

\section{Dovepress}

Journal Citation Reports/Science Edition, EMBase, Scopus and the Elsevier Bibliographic databases. The manuscript management system is completely online and includes a very quick and fair peer-review system, which is all easy to use. Visit http://www.dovepress.com/ testimonials.php to read real quotes from published authors. 